\title{
Technique for identifying cutting artefacts in sections of undecalcified bone biopsy specimens
}

\author{
D J Wood, W H B Mawhinney, A J Malcolm, J Stevens
}

\begin{abstract}
Areas of fragmentation found in trabecular and cortical bone of iliac crest biopsy specimens have been described as bone quality defects and were thought to be the major factor responsible for femoral neck fractures. These appearances have also been regarded as cutting artefacts and to resolve this difference of opinion consecutive sections cut at right angles to each other in biopsy specimens from 15 patients with femoral neck fractures were compared. Sections were assessed by four independent observers; agreement by at least three was required before an area was accepted as a bone quality defect. In all, 270 were identified. Of the 161 found in sections cut parallel to the cortices, there were only $20(12 \%)$ in coincident areas in consecutive sections. This study shows conclusively that areas of fragmentation previously described as bone quality defects are not artefacts which may be created or excluded depending on the plane of section.
\end{abstract}

Bone quality defects have been described as areas of fragmentation found in trabecular and cortical bone of iliac crest biopsy specimens from patients with femoral neck fractures (fig 1). It was concluded that they may be the major factor responsible for femoral neck fractures in the population aged over 60 years. ${ }^{1}$ These areas of fragmentation are not uncommon, and in our laboratory were always considered to be cutting artefacts. To resolve this difference of opinion, we compared consecutive sections cut at right angles to each other.

\section{Methods}

Iliac crest biopsy specimens were taken from 15 elderly patients undergoing surgery for subcapital femoral fracture. Standard $7 \mathrm{~mm}$ diameter biopsy specimens, including superficial and deep cortices and intervening trabecular bone, were harvested from a site $5 \mathrm{~cm}$ posterior and $5 \mathrm{~cm}$ inferior to the anterior superior iliac spine.

A modified block holder was used to hold the biopsy specimen level and was rotated through 90 degrees between sections. This modification of the standard Jung K orientating block holder has four equidistant stabilers set in the clamp resting on the circular base to ensure that the block face remains orientated in the same plane throughout rotation.

Specimens were fixed in absolute alcohol, dehydrated in Cellosolve, and embedded in the plastic resin Polymaster 1209AC. ${ }^{2}$ Undecalcified sections were cut at $6 \mu \mathrm{m}$ and the modified block holder was rotated between sections. Two sections from each biopsy specimen, cut at right angles to each other, were prepared for analysis. Consecutive sections were also cut without rotating the block, but the microtome blade was moved transversely by $1 \mathrm{~mm}$ between sections. Trabecular bone volume, osteoid volume, osteoid surface and osteoid index were determined, but did not differ from previously reported reference values. $^{3}$ Black and white photographs (magnification $\times 100$ ) were used as a tally sheet to record the site of bone quality defects seen on projected colour transparencies. Four independent observers, familiar with previously published work on bone quality defects, ${ }^{1}$ mapped out the sites, but agreement by at least three of the observers was required before an area was accepted as a bone quality defect. Photographs of consecutive sections were compared for coincident bone quality defects using a transparent grid overlay.

Of 161 bone quality defects (mean $10 \cdot 6 / \mathrm{sec}$ tion) observed in sections cut parallel to the cortices, only $20(12.4 \%)$ were coincident in sections cut at right angles to the cortices $(p<$ 0.001 , Mann Whitney $U$ test). Bone quality defects were frequently seen to disappear completely in consecutive sections in both cortical and trabecular bone (fig 2). A total of 270 were found, 161 in sections cut parallel to the 


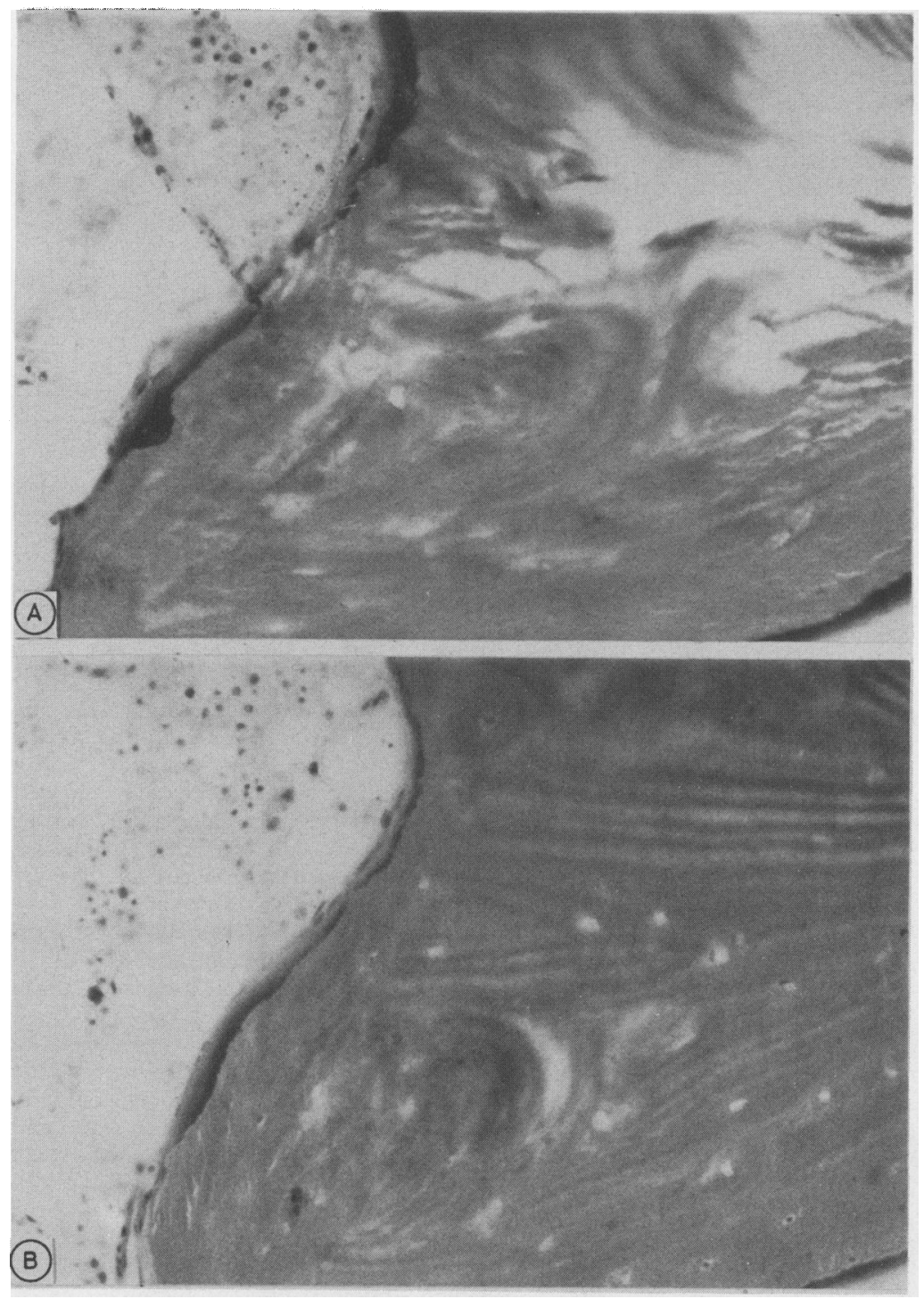

Figure 2 Bone quality defect in trabecular bone $(A)$ is not seen in the consecutive section $(B)$ cut at right angles to $(A)$. cortices and 109 in those cut perpendicular to the cortices. This difference was not significant and cannot be used as a guide to the optimal direction of section.

The use of this modified block holder has meant that previously described bone quality defects have been identified as cutting artefacts which may be created or excluded depending on the plane of section. These areas of fragmentation are not reliable indicators of the quality of underlying bone.

1 Ralis ZA. Bone weakness in the elderly. Hospital Update 1983:1381-91.

2 Mawhinney WHB, Ellis HA. A technique for plastic embedding of mineralised bone. J Clin Pathol 1983;37:1197-9. 3 Revell PA. Pathology of bone. Stuttgart: Springer-Verlag, 1985. 


\section{BPMF}

University of London

\section{British Postgraduate Medical Federation \\ Histopathology Course 1990-1991}

There will be a course for SHOs and registrars to provide training in histopathology for the new MRCPath Part 1 examination which begins in May 1992. It will be held on Wednesday afternoons at St Bartholomew's Hospital, and will consist of lectures and slide seminars with emphasis on diagnosis and mechanisms of disease.

The course will run from 3 October 1990-13 March 1991 and is the first in a series of three. The second and third courses will be offered from October 1991 and October 1992 respectively.

Places are restricted and early application is advised.

\section{Cost $£ 517$}

Application forms, which must be returned by 24 August 1990, may be obtained from:

The Education Department, BPMF, 33

Millman Street, London WC1N 3EJ. Telephone 071-831 6222 extension 155

\section{Association of Clinical Pathologists} Junior Membership

Junior membership of the Association is available to medical practitioners who have been engaged in the practice of pathology for a period of less than four years. Junior members are able to remain in this category for a maximum of six years or on the attainment of consultant status. The annual subscription is $£ 24$ for those resident in the United Kingdom and $£ 55$ for those overseas. The annual subscription may be claimed against tax.

Junior members receive the Journal of Clinical Pathology each month. Other benefits are reduced registration fees to attend ACP scientific meetings, all the documents regularly sent to full members of the Association including $A C P$ News, which has a regular column for juniors, and the twice yearly summary of pathology courses included in the ACP programme of postgraduate education. Junior members have their own representative body, the Junior Members' Group, which has a direct input to Council.

For Junior Membership apply to: The Honorary Secretary, Association of Clinical Pathologists, School of Biological Sciences, Falmer, Brighton, BN1 9QG. (0273) 678435.

\section{Second update in cardiopulmonary medicine}

6-8 November, 1990

A three day modular course designed for cardiologists, respiratory physicians, anaesthetics, pathologists, paediatricians and related scientists. Poster sessions will be a feature of the course.

\section{The Squibb Lecture}

Monday 5 November, 1990 at $5.30 \mathrm{pm}$ "Neuroendocrine control of cardiovascular and respiratory systems."

To be given by Professor Julia Polak followed by a champagne reception.

\section{Cardiology Day}

Tuesday 6 November, 1990

Cardiomyopathies-Hypertrophic cardiomyopathies, genetics, cardiomyopathies in fetal life, paediatric cardiomyopathies. Atrioventricular septal defect-Development and structure, deficient atrioventricular septation, atrioventricular conduction tissues, genetics, pulmonary circulation in AVSD.

\section{Cardiopulmonary Day}

Wednesday 7 November, 1990 Endothelin in the heart and lung-Expression in tissue and cell culture, effects: tissue selectivity and vascular activities of endothelin, the heart and coronary circulation, the microvasculature, renal failure, renal function, endothelin in the lung: distribution, receptors and pharmacology, bronchopulmonary actions of endothelin.

\section{Pulmonary Day}

Thursday 8 November, 1990 Cystic fibrosis-Genetics and molecular biology, membrane defect, autonomic innervation and receptor defects, pathological changes, clinical disease spectrum, long term respiratory problems, microbiology and treatment, energy expenditure and nutritional requirements, lung transplantation.

Adult respiratory distress syndromePathology, spectrum of the disease, ventilatory support, electrolyte disturbances, balance and nutrition, cardiovascular assessment and optimising oxygen delivery, recent advances in therapeutic intervention.

\section{Course Fee: $£ 150.00$ whole course} or $\mathbf{6 0 0 . 0 0}$ per day

Further details may be obtained from: The Postgraduate Education Centre, National Heart \& Lung Institute, Dovehouse Street, London SW3 6LY, UK. Tel: 071-351 8172 (24 $\mathrm{hr}$ answering service) Fax: 071-1376 3442
Senior Registrar Management Course, University of Keele

The next management course for senior registrars in pathology directed by Professor Roger Dyson at the University of Keele will be held from lunchtime on Wednesday 12 December, 1990 to lunchtime on Friday 14 December, 1990. If you wish to receive a copy of the programme and booking information for this course, please contact Tanya Matthews, (0782) 621111 ext 3646, or write to the Centre for Health Planning and Management, Suite 2.1, Science Park, University of Keele, Staffs ST5 5SP.

\section{Register of primary immune} deficiencies

In line with other European countries, a Register of all patients in the United Kingdom with primary immune deficiencies is being compiled. This is being organised by $\mathrm{Dr} \mathrm{J}$ Gooi (Immunology Department, Blood Transfusion Service, Bridle Path, Leeds LS15 7TW).

To gain complete coverage we should be grateful if any physicians or general practitioners, who have not already been contacted and who are currently managing such patients, could send details of their patients to Dr Gooi.

Registration forms are available from Dr J Gooi (0532 645091) or Dr H Chapel (0865 817305, Immunology Department, John Radcliffe Hospital, Oxford OX3 9DU).

\section{Corrections}

We regret that an error appeared in the Matters Arising "Screening for bacteriuria" 1989;42:557. The microscopy and culture method was described as costing $£ 0.90$; it should have read $£ 0.09$. Our apologies.

Apologies are extended to Dr G Markey for having changed the gender of the patient reported in his paper 1990;43:282 "Monocyte esterase deficiency in malignant neoplasia". The patient was female.

An error appeared in the last sentence of the paper by Wood et al "Technique for identifying cutting artefacts in sections of undecalcified bone biopsy specimens" 1990;43:516. "This study shows conclusively that areas of fragmentation previously described as bone quality defects are not artefacts which may be created or excluded depending on the plane of the section. They, of course, are artefacts. 